\title{
Assessment of thyroid function during pregnancy: the advantage of self-sequential longitudinal reference intervals
}

\author{
Qiu-wei Wang, Bin Yu, Rui-ping Huang, Fang Cao, Zi-qiang Zhu, Da-cheng Sun, Hong Zhou
}

Changzhou Women and Children Health Hospital to Nanjing Medical University, Jiangsu Province, China

Submitted: 30 October 2010

Accepted: 19 June 2011

Arch Med Sci 2011; 7, 4: 679-684

DOI: 10.5114/aoms.2011.24139

Copyright (c) 2011 Termedia \& Banach

\begin{abstract}
Introduction: To evaluate clinical value of a new self-sequential longitudinal reference intervals of thyroid function during pregnancy.

Material and methods: We established two different series of reference intervals: self-sequential longitudinal reference intervals (SLRI) and general gestationspecific reference intervals (GSRI). For SLRI, the serum of 301 cases were collected five times in every case throughout the gestation. For GSRI, A total of 1455 subjects included in the study. We collected the serum respectively at various trimesters. We used TSH of both reference intervals to screen 1744 pregnant women, and compared the percentage of potential misclassification.

Results: Both SLRI and GSRI differed substantially from that for non-pregnant women $(p<0.05)$. There are similar fluctuations of serum TSH, FT4 and TPO-Ab during normal pregnancy. Although there were no significant differences in most reference intervals between SLRI and GSRI. But the IQR of SLRI were usually smaller than GSRI, especially in $1^{\text {st }}$ trimester. Two hundred and fifty two women (14.4\%) at various trimesters whose serum TSH concentration was within SLRI would be misclassified, while 23 women $(1.3 \%)$ with a TSH concentration outside limit would not be identified. $0.11-3.84 \%$ women would got thyroid diseases during pregnancy. Subclinical hypothyroidism is most common maternal thyroid disorders.

Conclusions: The SLRI can reflected the changes of thyroid function realistically, and can be used to decrease the percentage of potential misclassification of thyroid dysfunction during pregnancy. Screening for thyroid dysfunction of pregnant women is recommended and important.
\end{abstract}

Key words: pregnancy, thyroid hormones, reference intervals, self-sequential, outcome.

\section{Introduction}

It is well known that it is very important to evaluate thyroid function accurately during pregnancy [1-3]. Maternal thyroid dysfunction or only the presence of thyroid-specific antibodies is associated with increased risk for early abortion, preterm delivery, neonatal morbidity [1, 4] and some obstetrical complications [5, 6], such as premature delivery, gestational hypertension, pre-eclampsia, and placental abruption. Benhadi et al. have reported that higher maternal TSH levels in pregnancy would increase miscarriage by $60 \%$ [7]. Männistö et al. found

\author{
Corresponding author: \\ Dr. Rui-ping Huang \\ Changzhou Women \\ and Children Health Hospital \\ Nanjing Medical University \\ Changzhou 213003 \\ No. 26 Bo Ai Road \\ Changzhou City 213003 \\ Jiangsu Province, China \\ E-mail: hrp117117@163.com
}


that women with thyroid dysfunction and antibodies during pregnancy were more prone to get thyroid disease later [8]. Maternal thyroid function is important not only to the parent but also to the fetus. For example, maternal hypothyroidism, even very mild, can have adverse effects on cognitive and neurological development of the fetus [2]. Both mild and severe maternal hypothyroxinaemia are a higher risk factor for cognitive delay in early childhood [9]. So recently researchers of perinatal medicine reached an agreement that screening thyroid dysfunction of pregnant women is recommended and important.

During a normal pregnancy, great physiological changes in the mother induce complex endocrine and immune responses. At the same time, thyroid gland volume may increase, the thyroid is hyperstimulated, and thyroid hormone concentrations change [10-13]. Recently, researchers recommended the use of gestation-specific reference intervals to evaluate maternal thyroid function. If a non-pregnant reference range is used, many maternal thyroid diseases could be potentially misclassified [14-16]. Some researchers have attempted to establish the gestation-specific reference intervals for a local population, such as Malaysian [16], Chinese [17, 18], Spanish [19], Indian [20], Australian [21], English [22], Czech [23], and so on. However, most studies are based on cross-sectional studies from different women in different stages of gestation. In our last study, we established new self-sequential longitudinal reference intervals based on the same group of pregnant women [24]. The new ranges could reflect the changes of thyroid function in pregnant women more realistically and reduce the variation caused by sampling error from different groups. Maybe it can also give a more accurate value for clinical diagnosis and therapy.

The aim of this study was to evaluate the clinical value of the self-sequential longitudinal reference intervals of thyroid function during pregnancy. First, we established two different series of reference intervals for serum thyroid stimulating hormone (TSH), free thyroxine (FT4) and thyroid peroxidase antibodies (TPO-Ab) during pregnancy: self-sequential longitudinal reference intervals (SLRI) and general gestation-specific reference intervals (GSRI). Then we used both reference intervals to clinically evaluate the status of thyroid function of pregnant women, and observed the percentage of potential misclassification and the relationship with pregnancy outcome. So we can know the advantage of the self-sequential longitudinal reference intervals for the diagnosis of thyroid dysfunction during pregnancy.

\section{Material and methods}

\section{Study design}

This study was designed and undertaken in the Changzhou Women and Children Health Hospital of Nanjing Medical University, Jiangsu Province, China. The cases of this study comprised the pregnant women who regularly checked in to our hospital for early pregnancy prenatal care from June 2008 to December 2009.

\section{Target population and inclusion criteria}

Based on the research of Teng [25], we established selection criteria as follows: 1 ) no past history of thyroid disease, 2) no life history in the areas of endemic goitre, 3) no other history of autoimmune diseases, 4) no medical history affecting thyroid function, 5) normal urinary iodine, 6) singleton pregnancies, 7) TPO-Ab negative $(<5.0$ $\mathrm{IU} / \mathrm{ml})$, 8) after follow-up, no hyperemesis gravidarum, pregnancy-induced hypertension, gestational diabetes, premature birth or other adverse maternal-fetal outcomes.

For SLRI, 301 cases were included in the study [24]. For GSRI, a total of 1455 subjects were included in the study: 406 in the $1^{\text {st }}$ trimester, 388 in the $2^{\text {nd }}$ trimester, and 661 in the $3^{\text {rd }}$ trimester were selected to establish general gestation-specific reference intervals. Their age was $26.21 \pm 6.35$ years and the height was $157.4 \pm 11.2 \mathrm{~cm}$.

\section{Samples collection}

For SLRI, samples of every case were collected five times throughout the gestation [24]. For GSRI, we collected the sera respectively from 1455 women at various trimesters of pregnancy. Three $\mathrm{ml}$ of blood of all the cases were collected by simple needle aspiration. The samples were centrifuged at $3000 \mathrm{rpm}$ for $5 \mathrm{~min}$ to remove cells. The serum was stored at $-86^{\circ} \mathrm{C}$ until assays.

\section{Test}

The levels of TSH, FT4 and TPO-Ab were quantified by electrochemistry immunoassay $(E C L)$ using a COBAS e601 automated analyser (Roche Diagnostics, Germany). We observed the dynamic change of thyroid hormone over all gestation. Then we established two reference intervals. All cases were followed by a survey of hospital medical record and telephone interview. The detection range of serum TSH is $0.005-100 \mathrm{mIU} / \mathrm{l}$, and $0.3-100$ $\mathrm{pmol} / \mathrm{l}$ for FT4, 5-600 IU/ml for TPO-Ab.

\section{Clinical evaluation}

In the study, we explored initially the value of clinical evaluation and diagnosis. Serum TSH 
provides the most sensitive index to reliably detect thyroid function abnormalities [26]. At first, we used TSH of both reference intervals to screen 1744 pregnant women (458 in the $1^{\text {st }}$ trimester, 449 in the $2^{\text {nd }}$ trimester, and 837 in the $3^{\text {rd }}$ trimester), and compared the percentage of potential misclassification. According to their gestational weeks, we selected the best reference intervals as the standard of diagnosis. After following up all cases, we observed the occurrences of pregnancy complication, including pre-eclampsia, hypertensive disorder complicating pregnancy, gestational diabetes mellitus, premature birth, intrahepatic cholestasis of pregnancy, and so on. Then, we analysed the relationship between misclassification and pregnancy outcome.

\section{Statistical analysis}

All data were collected and statistically analysed by SPSS 13.0 software. Results of parameters were expressed as median (M), interquartile range (IQR), $2.5^{\text {th }}$ percentile (P2.5) and 97.5 th percentile (P97.5). The limits of the reference intervals were calculated as P2.5 to P97.5. Non-parametric tests were employed to compare differences in thyroid hormones (the median level); comparisons among groups with different gestational ages were performed with Kruskal-Wallis test (H test) and comparisons between two different groups were compared with Wilcoxon test. Mann-Whitney U test was used to compare the difference between SLRI and GSRI. Positive ratios were calculated by $\chi^{2}$-test. A value of $p$ of less than 0.05 was considered to be statistically significant.

\section{Details of ethics approval}

The study design and protocol were reviewed and approved by the ethics committee of Changzhou Women and Children Health Hospital affiliated to Nanjing Medical University.

\section{Results}

Table I shows the median, P2.5-P97.5, IQR of serum TSH, FT4 and TPO-Ab. There are similar fluctuations of serum TSH, FT4 and TPO-Ab in SLRI and GSRI during normal pregnancy.

Compared with normal non-pregnant women, serum TSH was at a low level during the $1^{\text {st }}$ trimester $(p<0.05)$, and began to rise, but always at a lower level $(p<0.05)$ (Figure 1). The level of FT4 was consistently lower than the non-pregnant group $(p<0.05)$, and tended to a steady state (Figure 2). TPO-Ab rose continuously all over gestation. It was significantly higher in the $3^{\text {rd }}$ trimester $(p<0.05)$ (Figure 3$)$. There were no significant differences in most reference intervals between SLRI and GSRI. But the IQR of SLRI was usually smaller than GSRI, especially in the $1^{\text {st }}$ trimester.

Based on the results of 1744 pregnant women, SLRI had the best clinical specificity among three reference intervals in the $1^{\text {st }}$ trimester and $2^{\text {nd }}$ trimester. Women whose TSH level was outside SLRI would have higher risk of pregnancy complications $(p<0.01)$. The difference of risk was greatest in the $2^{\text {nd }}$ trimester $(34.6 \%$ vs. $9.5 \%$, $p<0.001)$. For GSRI, it was also satisfied results in the $2^{\text {nd }}$ trimester and $3^{\text {rd }}$ trimester $(p<0.05)$. However, there were no significant differences in non-pregnant reference interval throughout the gestation $(p>0.05)$.

In order to evaluate the value of clinical diagnosis, we investigated the thyroid function of 1744 pregnant women with SLRI. The number and percentage of potentially misclassification if nonpregnant reference intervals were used are summarized in Table II. Two hundred and fifty-two women (14.4\%) at various trimesters whose serum TSH concentration was within SLRI would be misclassified, while 23 women (1.3\%) with a TSH concentration outside the limit would not be

Table I. Reference intervals of thyroid function in normal pregnant women

\begin{tabular}{|c|c|c|c|c|c|c|c|c|}
\hline \multirow[t]{2}{*}{ Gestation } & \multirow[t]{2}{*}{ Group } & \multirow[t]{2}{*}{$n$} & \multicolumn{2}{|l|}{ TSH [mlU/l] } & \multicolumn{2}{|l|}{ FT4 [pmol/l] } & \multicolumn{2}{|c|}{ TPOAb [IU/ml] } \\
\hline & & & $\mathrm{M}\left(\mathrm{P}_{2.5}-\mathrm{P}_{97.5}\right)$ & IQR & $\mathrm{M}\left(\mathrm{P}_{2.5}-\mathrm{P}_{97.5}\right)$ & IQR & $\mathrm{M}\left(\mathrm{P}_{2.5}-\mathrm{P}_{97.5}\right)$ & IQR \\
\hline \multirow{2}{*}{$\begin{array}{l}1^{\text {st }} \\
\text { trimester }\end{array}$} & SLRI[24] & 301 & $1.00(0.02-3.65)^{\#}$ & 1.18 & 15.48 (11.85-21.51)\# & 3.05 & $6.35(5.00-19.69)^{*}$ & 4.03 \\
\hline & GSRI & 406 & $1.08(0.19-3.54)^{\#}$ & 1.26 & $16.04(12.01-24.62)^{\#}$ & 3.06 & $8.63(5.00-20.45)^{\#}$ & 6.83 \\
\hline \multirow{2}{*}{$\begin{array}{l}2^{\text {nd }} \\
\text { trimester }\end{array}$} & SLRI[24] & 301 & $1.26(0.36-3.46)^{\#}$ & 0.93 & $12.08(9.45-16.26)^{* \#}$ & 2.12 & $7.05(5.00-19.92)^{*}$ & 5.13 \\
\hline & GSRI & 388 & $1.35(0.38-3.29)^{\#}$ & 0.94 & $12.64(9.53-16.91)^{\#}$ & 2.46 & $9.57(5.00-31.05)^{\#}$ & 8.38 \\
\hline \multirow{2}{*}{$\begin{array}{l}3^{\text {rd }} \\
\text { trimester }\end{array}$} & SLRI[24] & 301 & $1.50(0.44-5.04)^{\star \#}$ & 1.10 & $12.62(9.30-17.14)^{\#}$ & 2.59 & $8.63(5.00-21.96)^{* \#}$ & 4.97 \\
\hline & GSRI & 661 & $1.72(0.51-5.43)^{\#}$ & 1.36 & 12.86 (9.37-17.14)\# & 2.52 & $9.71(5.00-25.68)^{\#}$ & 6.95 \\
\hline $\begin{array}{l}\text { Non-preg- } \\
\text { nancy[24] }\end{array}$ & & 150 & $2.10(0.75-5.28)$ & 1.35 & $17.71(14.35-21.69)$ & 2.22 & $6.78(5.00-22.42)$ & 2.92 \\
\hline
\end{tabular}

${ }^{*}$ Compared with GSRI group, $p<0.05,{ }^{*}$ Compared with non-pregnancy group, $p<0.05$ 


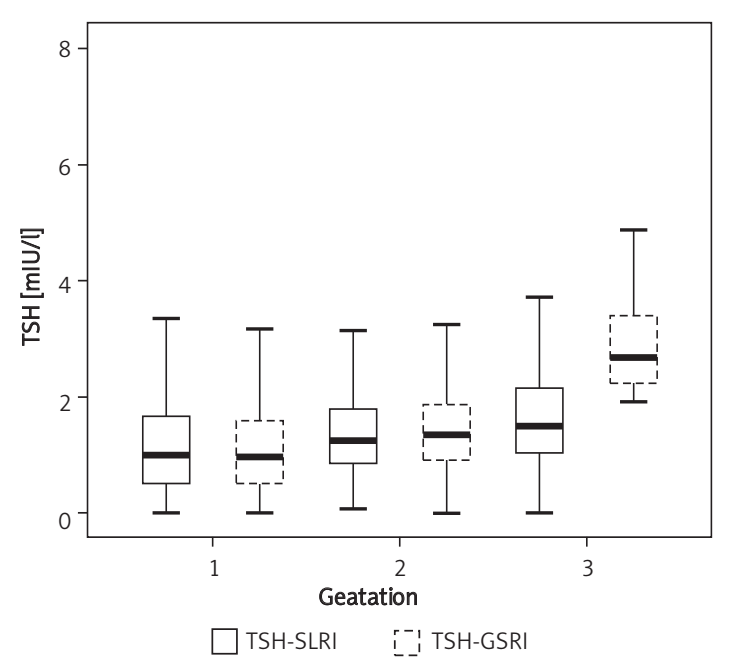

Figure 1. The fluctuations of both reference intervals of TSH in normal pregnancy

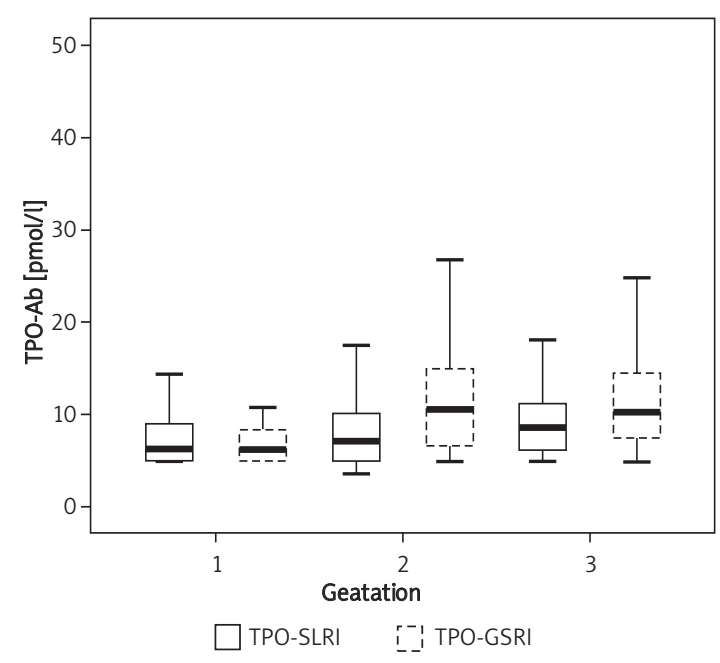

Figure 3. The fluctuations of both reference intervals of TPO-Ab in normal pregnancy

identified. The potential for misclassification of TSH results was greatest in the $1^{\text {st }}$ trimester $(31.0 \%)$. During the later pregnancy, $34.8 \%$ of missed diagnosis cases would have had some pregnancy complications. However, only $9.1 \%$ of the misdiagnoses might have occurred. If the GSRI was applied, a total of 40 women (2.3\%) would have

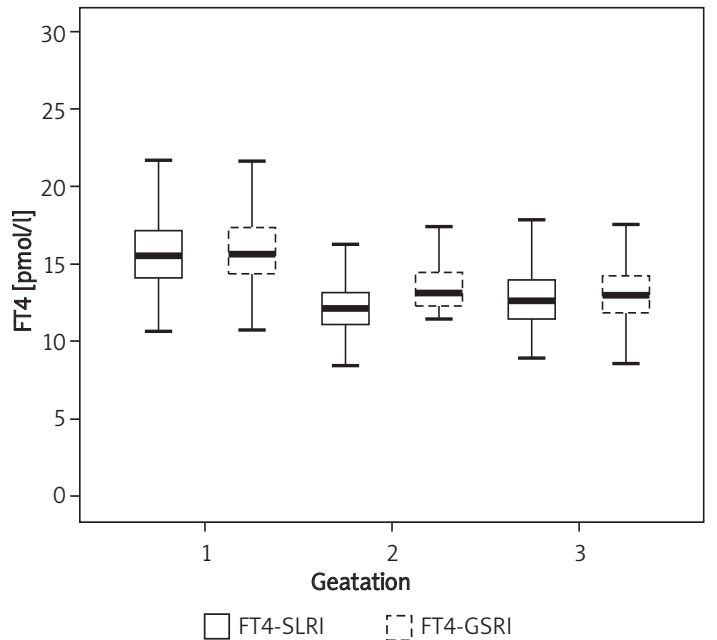

Figure 2. The fluctuations of both reference intervals of FT4 in normal pregnancy

been incorrectly classified, and only 3 women (0.1\%) would not have been identified. Both SLRI and GSRI were quite consistent in clinical diagnosis.

Table III shows the prevalence of thyroid hormone during pregnancy clinically evaluated with SLRI. 0.11-3.84\% of women would have got thyroid diseases during pregnancy, including hypothyroidism, subclinical hypothyroidism, and hyperthyroxinaemia. Subclinical hypothyroidism is the most common maternal thyroid disorder. The positive rate of TPO-Ab was $8.66 \%$ throughout the gestation.

\section{Discussion}

The basic premise for clinical management of thyroid disease in pregnancy is in accurate laboratory measurement of thyroid hormones. Because of complicated physiological changes during pregnancy, it is urged to establish the normal gestational-specific reference values of thyroid function. Recently, several studies have already established the normal gestational-specific reference intervals of thyroid function [16-23]. However, most of the present reference ranges were based on the cross-sectional survey from different populations. Andersen et al. [27] have reported that the intra-individual variation

Table II. Potential for misclassification of TSH in pregnant women using non-pregnant reference intervals

\begin{tabular}{|c|c|c|c|c|c|c|c|}
\hline \multirow[t]{2}{*}{ Gestation } & \multirow[t]{2}{*}{$N$} & \multicolumn{2}{|c|}{ Misdiagnosis } & \multicolumn{2}{|c|}{ Missed diagnosis } & \multicolumn{2}{|c|}{ Overall } \\
\hline & & $n(\%)$ & $\begin{array}{c}\text { Pregnancy } \\
\text { complication, } n(\%)\end{array}$ & $n(\%)$ & $\begin{array}{c}\text { Pregnancy } \\
\text { complication, } n(\%)\end{array}$ & $n(\%)$ & $\begin{array}{c}\text { Pregnancy } \\
\text { complication, } n(\%)\end{array}$ \\
\hline $1^{\text {st }}$ trimester & 458 & $134(29.3)$ & $13(9.7)$ & $8(1.7)$ & $2(25.0)$ & $142(31.0)$ & 15 (10.6) \\
\hline $2^{\text {nd }}$ trimester & 449 & $69(15.4)$ & $4(5.8)$ & 13 (2.9) & $6(46.2)$ & $82(18.3)$ & $10(12.2)$ \\
\hline $3^{\text {rd }}$ trimester & 837 & 49 (5.9) & $6(12.2)$ & $2(0.2)$ & 0 & $51(6.1)$ & $6(11.8)$ \\
\hline Total & 1744 & 252 (14.4) & $23(9.1)$ & $23(1.3)$ & $8(34.8)$ & $275(15.8)$ & 31 (11.3) \\
\hline
\end{tabular}


Table III. Prevalence of thyroid disorders during pregnancy using SLRI

\begin{tabular}{|lccccccc|}
\hline Gestation & $N$ & Hypothyroidism & $\begin{array}{c}\text { Subclinical } \\
\text { hypothyroidism }\end{array}$ & $\begin{array}{c}\text { Hypothyro- } \\
\text { xinaemia }\end{array}$ & $\begin{array}{c}\text { Hyperthyro- } \\
\text { xinaemia }\end{array}$ & $\begin{array}{c}\text { Subclinical } \\
\text { hyperthyro- } \\
\text { xinaemia }\end{array}$ & $\begin{array}{c}\text { TPO-Ab } \\
\text { positive }\end{array}$ \\
\hline $1^{\text {st trimester }}$ & 458 & $1(0.22)$ & $12(2.62)$ & $6(1.31)$ & $10(2.18)$ & $3(0.66)$ & $31(6.77)$ \\
\hline $2^{\text {nd }}$ trimester & 449 & $1(0.22)$ & $15(3.34)$ & $7(1.56)$ & $2(0.15)$ & $8(1.78)$ & $51(11.36)$ \\
\hline $3^{\text {rd trimester }}$ & 837 & 0 & $40(4.78)$ & $17(2.03)$ & $1(0.12)$ & $16(1.91)$ & $69(8.24)$ \\
\hline Total & 1744 & $2(0.11)$ & $67(3.84)$ & $30(1.72)$ & $13(0.75)$ & $27(1.55)$ & $151(8.66)$ \\
\hline
\end{tabular}

of thyroid function tests in non-pregnant individuals is smaller than the inter-individual variation. In our study, we established respectively two different series of reference intervals during pregnancy: selfsequential longitudinal reference intervals (SLRI) and general gestation-specific reference intervals (GSRI). Our study showed that both of them differed substantially from that for non-pregnant women. There were no significant differences in most reference intervals between SLRI and GSRI. But the IQR of SLRI was usually smaller than GSRI, especially in the $1^{\text {st }}$ trimester. So we considered that intraindividual variations of thyroid hormones were smaller than inter-individual variations also during pregnancy. Boas et al. [28] also drew a similar conclusion. The SLRI could reduce the variation caused by sampling error from different groups and reflected the changes of thyroid function in pregnant women more realistically, resulting in a more important value for clinical diagnosis and therapy.

More and more researchers are aware of the importance of evaluating maternal thyroid function during pregnancy by gestation-specific reference intervals. If a non-pregnant reference interval is used, a number of maternal thyroid diseases could be potentially misclassified. Stricker et al. [14] have reported that $5.6-18.3 \%$ of misdiagnoses and missed diagnoses likely occur in clinical practice due to the use of the non-pregnant normal population reference values as a basis for diagnosis. The potential for misclassification was greatest in the first trimester (10.4\%) for TSH [14]. In China, the percentage of potentially misclassified cases of subclinical hypothyroidism and hypothyroxinaemia in pregnant women was decreased by using the gestational age-specific reference intervals [15]. Similar results were obtained in Malaysia [16] and Australia [21]. As a single hormone determination, serum TSH provides the most sensitive index to reliably detect thyroid function abnormalities [26]. So we explored the clinical value of TSH of SLRI at first. We investigated the status of thyroid hormone by screening 1744 pregnant women with three kinds of reference intervals: SLRI, GSRI and non-pregnant reference intervals. After comparing the results and pregnancy outcome, we found that SLRI had the best clinical specificity among the three reference intervals. Women whose TSH level was outside SLRI would have higher risk of pregnancy complications, especially in the $1^{\text {st }}$ trimester and $2^{\text {nd }}$ trimester. Applying the non-pregnant reference intervals to pregnant women results in misclassification of thyroid status for $6.1-31.0 \%$ of women. Use of nonpregnant reference intervals has the potential to result in a large number of misclassified results. The SLRI can be used to decrease the percentage of potential misclassification of thyroid dysfunction.

Thyroid disorders are common in pregnancy and affect maternal and fetal outcome. Our data showed that $0.11-3.90 \%$ of women would have got thyroid diseases during pregnancy. Subclinical hypothyroidism is the most common maternal thyroid disorder. Some studies have reported that thyroid insufficiency may be associated with adverse obstetric outcome and fetal neurodevelopmental deficits $[1,29]$. So it is important to find thyroid hormone deficiency during the early stage of pregnancy. At the same time, TPO-Ab is used to predict some autoimmune thyroid diseases, such as post-partum thyroiditis [30]. Higher TPO-Ab level during pregnancy is also a marker of the danger of the development of postpartum thyroiditis [31]; about $50 \%$ of TPO-Ab positive women will get thyroid dysfunction after delivery [30-32]. In the present study, TPO-Ab was detectable in $8.66 \%$ of pregnant women. Evaluating the TPO-Ab levels may be helpful in later pregnancy. Overall, we think screening for thyroid dysfunction of pregnant women is recommended and important.

\section{Acknowledgments}

We thank all the study participants for their contributions. This study was supported by grants from Changzhou Health Bureau (WZ200821) and Key Laboratory of Changzhou City.

The authors declare that they have no competing interests.

RP Huang carried out the assays and participated in designing the study. B Yu, F Cao, ZQ Zhu, D Cheng, H Zhou carried out laboratory tests, participated in designing the study and performed the statistical analysis. RP Huang, B Yu, QW Wang conceived the study, participated in its design and coordination and helped draft the manuscript. 


\section{References}

1. Gärtner R. Thyroid diseases in pregnancy. Curr Opin Obstet Gynecol 2009; 21: 501-7.

2. Alexander EK. Thyroid function: the complexity of maternal hypothyroidism during pregnancy. Nat Rev Endocrinol 2009; 5: 480-1.

3. Galofre JC, Davies TF. Autoimmune thyroid disease in pregnancy: a review. J Womens Health 2009; 18: 1847-56.

4. Idris I, Srinivasan R, Simm A, et al. Maternal hypothyroidism in early and late gestation: effects on neonata and obstetric outcome. Clin Endocrinol 2005; 63: 560-5.

5. Glinoer D. Feto-maternal repercussions of iodine deficiency during pregnancy. An update. Ann Endocrinol 2003; 64: 37-44.

6. Luton D, Le Gac I, Noel M, et al. Thyroidfunction during pregnancy in women with past Graves disease. BJOG 2005; 112: 1565-7.

7. Benhadi N, Wiersinga WM, Reitsma JB, et al. Higher maternal TSH levels in pregnancy are associated with increased risk for miscarriage, fetal or neonatal death. Eur J Endocrinol 2009; 160: 985-91.

8. Männistö T, Vääräsmäki $M$, Pouta $A$, et al. Thyroid dysfunction and autoantibodies during pregnancy as predictive factors of pregnancy complications and maternal morbidity in later life. J Clin Endocrinol Metab 2010; 95: 1084-94.

9. Henrichs J, Bongers-Schokking JJ, Schenk JJ, et al. Maternal thyroid function during early pregnancy and cognitive functioning in early childhood: the generation R study. J Clin Endocrinol Metab 2010; 95: 4227-34.

10. Casey BM, Leveno KJ. Thyroid disease in pregnancy. Obstet Gynecol 2006; 108: 1283-92.

11. Panesar NS, Li CY, Rogers MS. Reference intervals for thyroid hormones in pregnant Chinese women. Ann Clin Biochem 2001; 38: 329-32.

12. Pearce EN, Oken E, Gillman MW, et al. Association of firsttrimester thyroid function test values with thyroperoxidase antibody status, smoking, and multivitamin use. Endocr Pract 2008; 14: 33-9.

13. Fister P, Gaberscek S, Zaletel K, et al. Thyroid volume changes during pregnancy and after delivery in an iodinesufficient Republic of Slovenia. Eur J Obstet Gynecol Reprod Biol 2009; 145: 45-8.

14. Stricker R, Echenard M, Eberhart R, et al. Evaluation of maternal thyroid function during pregnancy: the importance of using gestational age-specific reference intervals. Eur J Endocrinol 2007; 157: 509-14

15. Shan ZY, Chen Y, Teng WP, et al. A study for maternal thyroid hormone deficiency during the first half of pregnancy in China. Eur J Clin Invest 2009; 39: 37-42.

16. Thevarajah M, Chew YY, Lim SC, et al. Determination of trimester specific reference intervals for thyroid hormones during pregnancy in Malaysian women. Malays J Pathol 2009; 31: 23-7.

17. Yan Yu-qin, Dong Zuo-liang, Dong Ling, et al. Trimesterspecific reference data of thyroid hormones for normal pregnancy. Chin J Endocrinol Metab 2008; 24: 609-12.

18. Li Jia, Teng Wei-ping, Shan Zhong-yan, et al. Gestational month-specific reference ranges for TSH and thyroxine in Han nationality women in iodine sufficient area of China. Chin J Endocrinol Metab 2008; 24: 605-8.

19. Bocos-Terraz JP, Izquierdo-Alvarez S, Bancalero-Flores JL, et al. Thyroid hormones according to gestational age in pregnant Spanish women. BMC Res Notes 2009; 26: 237-46.
20. Marwaha RK, Chopra S, Gopalakrishnan S, et al. Establishment of reference range for thyroid hormones in norma pregnant Indian women. BJOG 2008; 115: 602-6.

21. Gilbert RM, Hadlow NC, Walsh JP, et al. Assessment of thyroid function during pregnancy: first-trimester (weeks 9-13) reference intervals derived from Western Australian women. Med J Aust 2008; 189: 250-3.

22. Cotzias C, Wong SJ, Taylor E, et al. A study to establish gestation-specific reference intervals for thyroid function tests in normal singleton pregnancy. Eur J Obstet Gynecol Reprod Biol 2008; 137: 61-6.

23. Springer D, Zima T, Limanova Z. Reference intervals in evaluation of maternal thyroid function during the first trimester of pregnancy. Eur J Endocrinol 2009; 160: 791-7.

24. Bin Yu, Qiu-wei Wang, Rui-ping Huang, et al. Establishment of a self-sequential longitudinal reference intervals of maternal thyroid function during pregnancy. Exp Biol Med 2010; 235: 1212-5.

25. Teng W, Shan Z, Teng X, et al. Effect of iodine intake on thyroiddiseases in China. N Engl J Med 2006; 354: 2783-93.

26. Glinoer D, Spencer CA. Serum TSH determinations in pregnancy: how, when and why? Nat Rev Endocrinol 2010; 6: 526-9.

27. Andersen S, Pedersen KM, Bruun NH, Laurberg P. Narrow individual variations in serum T(4) and T(3) in normal subjects: a clue to the understanding of subclinical thyroid disease. J Clin Endocrinol Metab 2002; 87: 1068-72.

28. Boas M, Forman JL, Juul A, et al. Narrow intra-individual variation of maternal thyroid function in pregnancy based on a longitudinal study on 132 women. Eur J Endocrinol 2009; 161: 903-10.

29. Soldin OP. Thyroid function testing in pregnancy and thyroid disease: trimester-specific reference intervals. Ther Drug Monit 2006; 8: 8-11.

30. Filippi U, Brizzolara R, Venuti D, et al. Prevalence of postpartum thyroiditis in Liguria (Italy): an observational study. J Endocrinol Invest 2008; 31: 1063-8.

31. Nicholson WK, Robinson KA, Smallridge RC, et al. Prevalence of postpartum thyroid dysfunction: a quantitative review. Thyroid 2006; 16: 573-82.

32. Premawardhana LD, Parkes AB, John R, et al. Thyroid peroxidase antibodies in early pregnancy: utility for prediction of post partum thyroid dysfunction and implications for screening. Thyroid 2004; 14: 610-5. 